\begin{tabular}{|c|c|c|c|c|c|c|c|c|c|}
\hline \multirow{2}{*}{\multicolumn{2}{|c|}{$\frac{1900}{\text { Sett. } 18}$}} & \multicolumn{3}{|c|}{$\alpha$ vera } & \multicolumn{3}{|c|}{$\delta$ vera } & \multirow{3}{*}{$\frac{\log \Delta}{\begin{array}{r}9.823483 \\
8183^{2} 5\end{array}}$} & \multirow{2}{*}{$\frac{\text { t. di ab. }}{5^{m} 3^{2^{s}}}$} \\
\hline & & \multicolumn{3}{|c|}{$2^{h} 3^{8^{m}} 21^{3} \cdot 3^{8}$} & $+40^{\circ}$ & \multicolumn{2}{|c|}{ 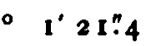 } & & \\
\hline & 19 & & 39 & $3.3^{\circ}$ & 40 & 24 & 13.9 & & 28 \\
\hline & 20 & & 39 & 42.89 & 40 & 47 & 7.2 & 813.57 & 24 \\
\hline & 21 & & 40 & 20.04 & 41 & 10 & 0.9 & 807982 & 20 \\
\hline & 22 & & 40 & 54.69 & $4 I$ & $3^{2}$ & 54.4 & 802800 & 16 \\
\hline & 23 & & 41 & 26.74 & 41 & 55 & $47 \cdot 4$ & 797612 & 13 \\
\hline & 24 & & 41 & $5^{6.12}$ & 42 & I 8 & 39.2 & $79242 \mathrm{I}$ & 9 \\
\hline & 25 & & $4^{2}$ & 22.75 & 42 & 41 & $29 \cdot 4$ & 787226 & 5 \\
\hline & 26 & & 42 & 46.55 & 43 & 4 & 17.1 & 782030 & 2 \\
\hline & 27 & & 43 & $7 \cdot 41$ & 43 & 27 & 2.0 & 776834 & $45^{8}$ \\
\hline & 28 & & 43 & 25.24 & 43 & 49 & 43.2 & 771640 & 54 \\
\hline & 29 & & 43 & 40.01 & 44 & 12 & 20.4 & 766448 & 51 \\
\hline & 30 & & 43 & $5^{1.63}$ & 44 & 34 & 52.7 & 761260 & 48 \\
\hline Ott. & $\mathbf{I}$ & & 43 & 59.96 & 44 & 57 & 189 & 756078 & 44 \\
\hline & 2 & & 44 & 4.92 & 45 & I 9 & 38.2 & 750903 & $4 I$ \\
\hline & 3 & & 44 & 6.44 & 45 & $4 I$ & 50.2 & 745736 & 38 \\
\hline & 4 & & 44 & $4 \cdot 44$ & 46 & 3 & 5.3 .8 & 740578 & 34 \\
\hline & 5 & & 43 & 58.85 & 46 & 25 & 48.2 & 735431 & 31 \\
\hline & 6 & & 43 & 49.57 & 46 & 47 & 32.2 & 730297 & 28 \\
\hline & 7 & & 43 & $3^{6.50}$ & 47 & 9 & 5.1 & 725177 & 25 \\
\hline & 8 & & 43 & 19.53 & 47 & 30 & 25.4 & 720073 & 22 \\
\hline & 9 & 2 & 42 & $5^{8} \cdot 5^{6}$ & +47 & $5^{I}$ & 32.1 & 9.714984 & 419 \\
\hline
\end{tabular}

$$
1900
$$

$$
\begin{aligned}
& \text { Sett. I. } 5 \\
& \text { Ott. } 11.5 \\
& 31.5
\end{aligned}
$$

\begin{tabular}{|c|c|c|c|c|c|c|c|c|}
\hline 1900 & \multicolumn{2}{|c|}{$\alpha$ vera } & \multicolumn{3}{|c|}{$\delta$ vera } & $\log \Delta$ & \multicolumn{2}{|c|}{ t. di ab. } \\
\hline Jtt. 10 & $2^{h} 42^{r}$ & $33^{5} \cdot 51$ & $+48^{\circ}$ & I $2^{\prime}$ & $23: 6$ & 9.709914 & & ${ }^{n} 16^{5}$ \\
\hline I I & 42 & 4.23 & 48 & $3^{2}$ & 58.9 & 704864 & & I 3 \\
\hline 12 & 41 & 30.67 & 48 & 53 & 163 & 699836 & & Io \\
\hline 13 & 40 & 52.76 & 49 & 13 & 14.2 & $694^{832}$ & & 7 \\
\hline 14 & 40 & 10.47 & 49 & $3^{2}$ & 50.9 & 689854 & & 4 \\
\hline 15 & 39 & 23.66 & 49 & $5^{2}$ & $5 \cdot 1$ & 684906 & 4 & 1 \\
\hline 16 & $3^{8}$ & $32 \cdot 33$ & 50 & 10 & 54.8 & 679988 & 3 & $5^{8}$ \\
\hline 17 & 37 & 36.44 & 50 & 29 & 18.2 & 675103 & & $5^{6}$ \\
\hline 18 & 36 & 36.00 & 50 & 47 & 13.4 & 670253 & & 53 \\
\hline 19 & 35 & 30.95 & 51 & 4 & $3^{8.6}$ & 66544 I & & $5 !$ \\
\hline 20 & 34 & $21 \cdot 37$ & $5 \mathrm{I}$ & 21 & 32.0 & 660668 & & 48 \\
\hline $2 \mathrm{I}$ & 33 & 7.26 & $5^{I}$ & 37 & 51.5 & 655939 & & 45 \\
\hline 22 & 31 & 48.74 & 51 & 53 & 35.1 & 651254 & & 43 \\
\hline 23 & 30 & 25.82 & 52 & 8 & 41.0 & 6466 I5 & & $4 I$ \\
\hline 24 & 28 & 58.65 & $5^{2}$ & 23 & $7 \cdot 4$ & 642026 & & $3^{8}$ \\
\hline 25 & 27 & $27 \cdot 35$ & $5^{2}$ & 36 & 52.0 & 637488 & & 36 \\
\hline 26 & 25 & 52.13 & $5^{2}$ & 49 & 53.2 & 633004 & & 34 \\
\hline 27 & 24 & 13.05 & 53 & 2 & 9.2 & 628575 & & $3^{2}$ \\
\hline 28 & 22 & 30.39 & 53 & 13 & 38.2 & 624203 & & 30 \\
\hline 29 & 20 & $44 \cdot 30$ & 53 & 24 & 18.5 & 619890 & & 28 \\
\hline 30 & 18 & 55.10 & 53 & 34 & 8.5 & $615^{6} 37$ & & 26 \\
\hline $3 I$ & 217 & 2.99 & +53 & 43 & 6.5 & $9.61144^{8}$ & 3 & 24 \\
\hline
\end{tabular}

\begin{tabular}{|c|c|c|c|c|c|c|c|}
\hline \multicolumn{2}{|c|}{1900} & $\log r$ & 1900 & $\log r$ & \multicolumn{2}{|c|}{1900} & $\log r$ \\
\hline Sett. & 1.5 & $0.18295^{6}$ & Sett. $17 \cdot 5$ & 0.168777 & Ott. & $3 \cdot 5$ & 0.153484 \\
\hline & $5 \cdot 5$ & 0.179525 & 21.5 & 0.165051 & & $7 \cdot 5$ & 0.14951 \\
\hline & 9.5 & 0.176017 & $25 \cdot 5$ & $0.161257^{\circ}$ & & 11.5 & 0.145492 \\
\hline & 13.5 & 0.172434 & 29.5 & $0.15740 \mathrm{r}$ & & 15.5 & 0.14142 \\
\hline
\end{tabular}

$$
\begin{gathered}
M \text { (per osser- } \\
\text { vazione diretta) }
\end{gathered}
$$

$$
\begin{array}{r}
11.06 \\
10.46 \\
9.85 \\
9.28
\end{array}
$$

Roma, I90o Agosto 14 .

$M$ fotografica tenuto conto di fase (A.N. 3637)

$$
\begin{aligned}
& 12.33 \\
& 11.65 \\
& 10.93 \\
& 10.29
\end{aligned}
$$$$
\text { (1) }
$$

$$
\text { (1) }
$$

PS. Dal mio amico, Prof. Abetti, direttore dell' osservatorio di Arcetri, ho ricevuto in ritardo per usare nej calcoli, una bella serie di osservazioni di (433) Eros, in numero di 22.

Estraendo da essa le osservazioni fra Luglio 25 e Luglio $3 \mathrm{I}$, in numero di ${ }_{13}$, ebbi il seguente luogo normale:

Coi miei nuovi elementi si ha:

$$
\text { 1900.0 } \alpha=22^{\circ} 5^{\prime} 4^{\prime \prime}{ }^{\prime} 5^{6} \delta=+21^{\circ} 32^{\prime} 26^{\prime \prime} 4^{2} \quad \text { 1900 Lugl. } 28.5 \text { Berlino. }
$$

$$
\begin{array}{r}
1900.0 \alpha=22^{\circ} 5^{\circ} 49^{\prime \prime} .54 \quad \delta=+21^{\circ} 3^{2} 28.21 \\
\mathrm{O}-\mathrm{C}=-0^{\prime \prime} .98
\end{array}
$$

I valori trovati tranquillizzano sull' esattezza del luogo normale utilizzato nella correzione degli elementi, e sui procedimenti di conteggio.

E. $M$.

\title{
Aufsuchungs-Ephemeride für den Cometen 1884 II (Barnard).
}

\section{Von A. Berberich.}

Der periodische Comet $\mathbf{1 8 8}_{4}$ II (Barnard) würde am 28. October igoo sein Perihel passiren, falls die in A.N. Bd. 123 p. 175 mitgetheilten Elemente richtig sind. Bei den Periheldurchgängen von 1890 und 1895 wurde der Comet wegen ungunstiger Stellung und Lichtschwăche nicht aufgefunden. Auch in diesem Jahre sind die Sichtbarkeitsbedingungen sehr unguinstig. Trotzdem wäre es wünschenswerth, dass wenigstens einige Versuche mit lichtstarken Fernrohren 
und photographischen Instrumenten gemacht wurden, den Cometen jetzt wiederzufinden. Denn im Jahre 1906 wird er in den Sonnenstrahlen verborgen bleiben und wahrend des folgenden Umlaufs werden erhebliche Jupiterstörungen die Vorausberechnung für spătere Wiederkunfte zur Unmöglichkeit machen. Die der berechneten Umlaufszeit anhaftende Unsicherheit kann schon in der angegebenen Zeit des nächsten Periheldurchgangs einen Fehler von vielleicht zwei bis drei Wochen bewirken. Doch hat dieser Fehler auf den Ort des Conseten gegenwärtig nur einen mässigen Einfluss. Ich gebe hier drei Ephemeriden; die mittlere entspricht dem wahrscheinlichsten Datum des Periheldurchgangs, während die anderen mit einer Variation der mittleren Anomalie um $\mp 16 \mu$ berechnet sind. Die unter genäherter Berücksichtigung der Jupiterstörungen abgeleiteten Elemente lauten:
Epoche 1900 Juli 13.0 M. Z. Berlin.

$$
\begin{aligned}
& M=340^{\circ} 25^{\circ} 7^{\prime \prime} \\
& \infty=30054 \quad 3 \\
& \delta=\begin{array}{lll}
520 & 20 \\
5 & 2000.0
\end{array} \\
& i=52731 \\
& \varphi=353917 \\
& \mu=655^{\prime \prime 17} \\
& \log a=0.48910
\end{aligned}
$$

Die Helligkeit, berechnet nach der Formel $I=\mathrm{I}: r^{2} \Delta^{2}$, bleibt durchweg geringer als bei der letzten Beobachtung am 20. November 1884 . Indessen ist eine vermehrte Lichtstärke um die Zeit des Perihels nicht unwahrscheinlich, die Wahrnehmbarkeit des Cometen also nicht völlig ausgeschlossen.

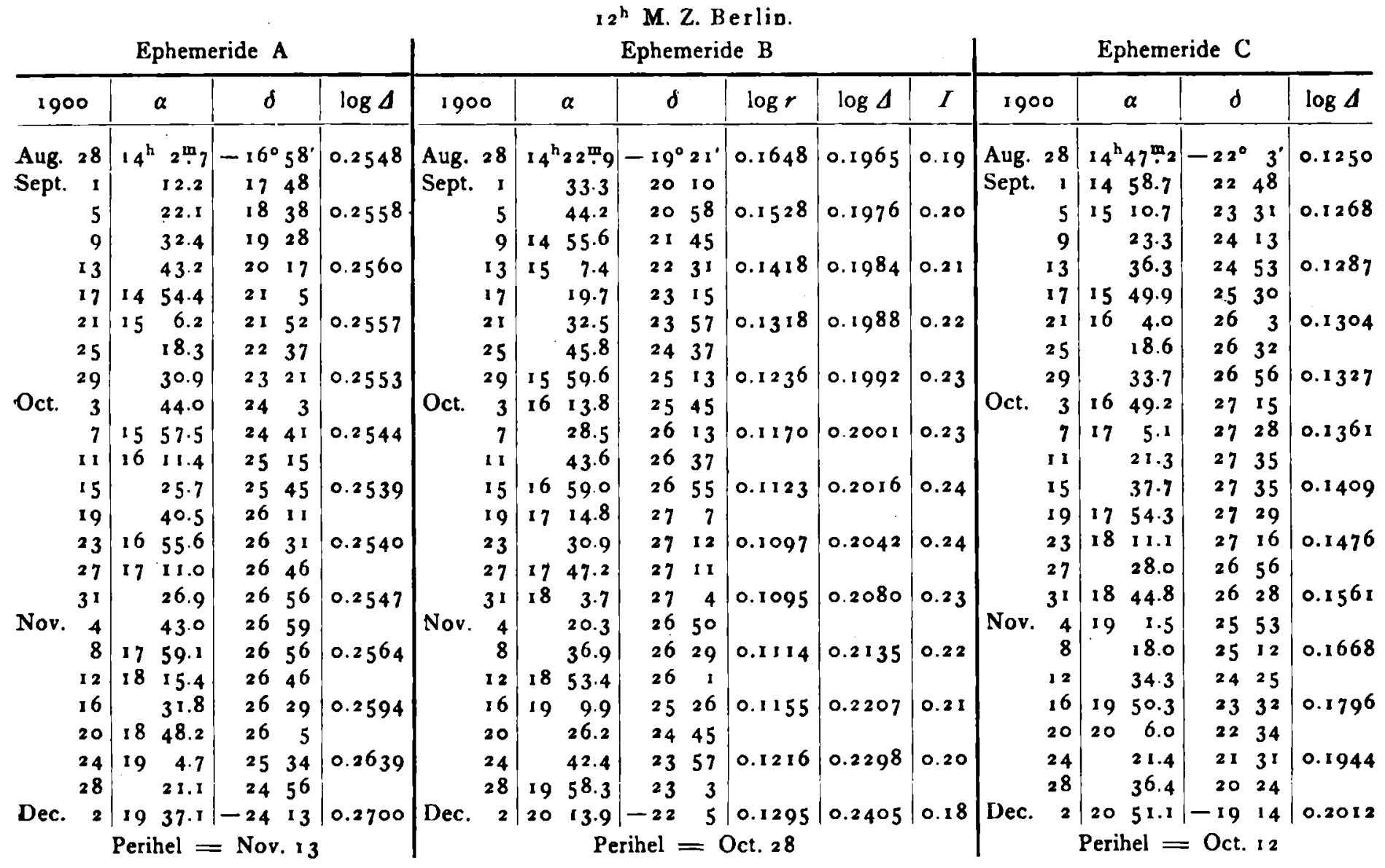

Berlin, 1900 Aug. 18.

A. Berberich.

\section{Elemente und Ephemeride des Cometen 1900 b.}

Aus den Beobachtungen Paris Juli 24 , Hamburg Juli 3 I und Aug. 7. ergeben sich folgende Elemente:

$$
\left.\begin{array}{rl}
T & =1900 \text { Aug. } 3.23^{6} 5^{\mathrm{I}} \text { M. Z. Berlin } \\
w & =12^{\circ} 25^{\circ} 34.8 \\
\delta & =328 \text { 0 } 26.2 \\
i & =623044.0
\end{array}\right\} 1900.0
$$

Darstellung des mittleren Ortes.

$\Delta \alpha \cos \delta=-3.0, \Delta \delta=-1.0$.

Heliocentrische Aequatorealcoordinaten.

$$
\begin{aligned}
& x=[9.945797] r \cdot \sin \left(v+86^{\circ} 20^{\circ} 32^{\prime \prime} 4\right) \text {. } \\
& y=[9.686710] r \cdot \sin (v+283825.9) \\
& z=[9.996635] r \cdot \sin (v+0.927 .4)
\end{aligned}
$$

